\title{
Semi-empirical formulas of drag/lift coefficients for high speed rigid body manoeuvring in water column
}

\author{
P. C. $\mathrm{Chu}^{1}$, C. Fan $^{1} \&$ P. R. Gefken ${ }^{2}$ \\ ${ }^{I}$ Naval Ocean Analysis and Prediction Laboratory, \\ Naval Postgraduate School, Monterey, California, USA \\ ${ }^{2}$ Polter Laboratory, SRI International, Menlo Park, California, USA
}

\begin{abstract}
Falling of rigid body through water column with high speed is investigated experimentally and theoretically. Several experiments were conducted to shoot rigid bodies with the density ratio higher than 1 into the hydrographical tank. During the experiments, we carefully observe the position and orientation of the bomb-like rigid bodies. Using the experimental data a semi-empirical formulas for the drag/lift coefficients were obtained. With the given drag/lift coefficients, the momentum and moment of momentum equations of a fast-moving rigid body can be integrated numerically. The numerical results are well compared by the experimental data.
\end{abstract}

Keywords: body-flow interaction, bomb manoeuvring, body trajectory and orientation, drag/lift coefficients, inverse modeling, bomb drop experiments.

\section{Introduction}

Study on falling rigid body through water column with high water entry speed has wide scientific significance and technical application. Scientific studies involve nonlinear dynamics, body and multi-phase fluid interaction, supercavitation, bubble dynamics, and instability theory. Technical application of the hydrodynamics of a rigid body moving fast in fluids includes aeronautics, navigation, and civil engineering.

The rigid-body dynamics allows one to set up six nonlinear equations for the most general motion (Chu et al., 2004, 2005a, b): three momentum equations and 
three moment-of-momentum equations. These equations are ready to solve if hydrodynamic coefficients (such as drag and lift coefficients) are given for computing hydrodynamic forces and torques. Unfortunately, these coefficients (usually Reynolds number dependent) are known only for bodies with some simple geometry such as cylindrical and spherical, not for bodies with more complicated geometry such as the Mk-84 general purpose bombs. Therefore, the key issue for solving the basic equations is to determine the hydrodynamic coefficients.

In this study, an inverse model is developed for determining the drag/lift coefficients from the rigid-body's trajectory and orientation. Then a bomb strike experiment is conducted to collect the data for the trajectory and orientation. Using the experimental data, semi-empirical formulas are derived for the drag/lift coefficients.

\section{Hydrodynamic force and torque}

Consider an axially symmetric rigid-body with length $L$ such as bomb falling through water column with the centers of mass $\left(c_{m}\right)$ and volume $\left(c_{v}\right)$ on the main axis (Fig. 1a). The position of the body is represented by the position of $c_{m}$,

$$
\mathbf{r}(t)=x \mathbf{i}+y \mathbf{j}+z \mathbf{k},
$$

which is called the translation. The positions of the two end-points (such as head and tail points) are represented by $\mathbf{r}_{h}(t)$ and $\mathbf{r}_{t}(t)$. The difference between the two vectors in nondimensional form

$$
\mathbf{e}=\frac{\mathbf{r}_{h}-\mathbf{r}_{t}}{\left|\mathbf{r}_{h}-\mathbf{r}_{t}\right|},
$$

is the unit vector representing the body's main axis direction. The translation velocity is given by

$$
\frac{d \mathbf{r}}{d t}=\mathbf{u}, \quad \mathbf{u}=V \mathbf{e}_{v} .
$$

where $V$ and $\mathbf{e}_{v}$ are the speed and unit vector of the rigid-body velocity.

Let $\mathbf{v}$ be the water-to-body relative velocity (called the relative velocity). If the water velocity is much smaller than the rigid-body velocity, the water-tobody relative velocity can be approximately given by

$$
\mathbf{v} \approx-\mathbf{u}=-V \mathbf{e}_{v}
$$

Usually the two vectors $\left(\mathbf{e}, \mathbf{e}_{v}\right)$ are not parallel and their vector product leads to a unit attack vector

$$
\mathbf{e}_{\alpha}=\frac{\mathbf{e}_{v} \times \mathbf{e}}{\sin \alpha}
$$

where $\alpha$ is the angle between $\left(\mathbf{e}, \mathbf{e}_{v}\right)$. For a two dimensional motion, if $(\beta, \gamma)$ are the elevation angles of the rigid body and its velocity, the difference $\alpha=\beta-\gamma$, is the water attack angle (Fig. 1b). 
Hydrodynamic force on a rigid body consists of a drag force $\left(\mathbf{F}_{d}\right)$

$$
\mathbf{F}_{d}=f_{d} \mathbf{e}_{d}, \mathbf{e}_{d}=-\mathbf{e}_{v},
$$

and a lift force $\left(\mathbf{F}_{l}\right)$

$$
\mathbf{F}_{l}=f_{l} \mathbf{e}_{l}, \mathbf{e}_{l}=\frac{\left(\mathbf{e}_{v} \times \mathbf{e}\right) \times \mathbf{e}_{v}}{\left|\left(\mathbf{e}_{v} \times \mathbf{e}\right) \times \mathbf{e}_{v}\right|} .
$$

Their magnitudes are determined by the drag and lift laws,

$$
f_{d}=\frac{1}{2} C_{d} \rho A_{w} V^{2}, f_{l}=\frac{1}{2} C_{l} \rho A_{w} V,
$$

where $\rho$ is the water density; $A_{w}$ is the under-water area of projection; and $\left(C_{d}, C_{l}\right)$ are the drag and lift coefficients. Let $\boldsymbol{\sigma}$ be the vector from the center of mass $(\mathrm{COM})$ to the center of volume (COV), or $\boldsymbol{\sigma}=\sigma \mathbf{e}$. The center of the hydrodynamic force (COF) is the location where the fulcrum is chosen with zero torque. In fact, the hydrodynamic torque is calculated from the resultant drag and lift force exerted on COF with COM as the fulcrum $\left(\mathbf{M}_{h}^{*}\right)$. If we shift the exerted point of the resultant drag and lift force from $\mathrm{COF}$ to $\mathrm{COV}$, the torque $\left(\mathbf{M}_{h}^{*}\right)$ contains two parts

$$
\mathbf{M}_{h}^{*}=\mathbf{M}_{h}+\mathbf{M}_{t}, \quad \mathbf{M}_{h}=\sigma \mathbf{e} \times\left(\mathbf{F}_{d}+\mathbf{F}_{l}\right), \quad \mathbf{M}_{t}=M_{t} \mathbf{e}_{\alpha},
$$

where $\mathbf{M}_{h}$ is the hydrodynamic torque with the resultant drag and lift force exerted on COV, and $\mathbf{M}_{t}$ is the torque caused by the shift of the exerting point from COF to COV. Here, the magnitude of $\mathbf{M}_{t}$ is calculated by the drag law

$$
M_{t}=\frac{1}{2} C_{m} \rho A_{w} L_{w} V^{2},
$$

where $C_{m}$ is the moment coefficient; and $L_{w}$ is the under-water path length.

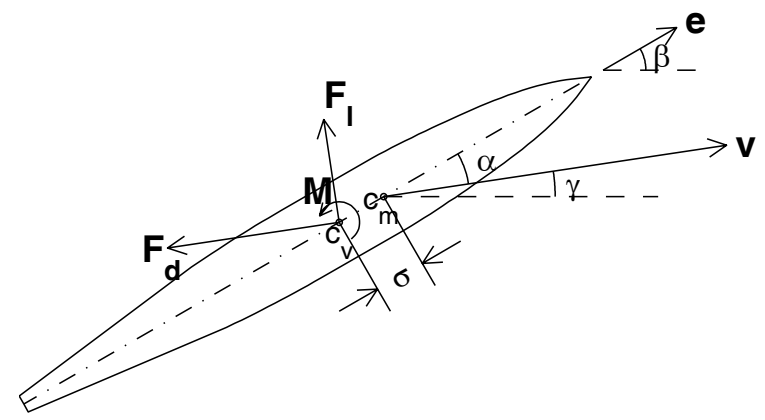

Figure 1: $\quad$ Shift of the exerted point of the drag/lift forces from COF to COV with an extra torque $\mathrm{M}_{\mathrm{t}}$. Here, $(\alpha, \beta, \gamma)$ are the attack angle, elevation angels of the body and its velocity. 


\section{Basic equations}

Let $(\mathbf{i}, \mathbf{j}, \mathbf{k})$ be the unit vectors of the Cartesian coordinate system fixed to the Earth with $(\mathbf{i}, \mathbf{j})$ in the horizontal plan and $\mathbf{k}$ in the vertical (positive upward). Time derivative of (2a) gives the acceleration of COM (Chu et al., 2006a, b),

$$
\frac{d \mathbf{u}}{d t}=\frac{d V}{d t} \mathbf{e}_{v}+V \frac{d \mathbf{e}_{v}}{d t} .
$$

If the translation velocity has an elevation angle $\gamma$ and an azimuth angle $\psi$, the unit vector $\mathbf{e}_{v}$ is represented by

$$
\mathbf{e}_{v}=\cos \gamma \cos \psi \mathbf{i}+\cos \gamma \sin \psi \mathbf{j}+\sin \gamma \mathbf{k},
$$

Substitution of (10) into (9) leads to

$$
\frac{d \mathbf{e}_{v}}{d t}=\frac{d \gamma}{d t} \mathbf{e}_{v}^{\gamma}+\frac{d \psi}{d t} \mathbf{e}_{v}^{\mu},
$$

where

$$
\begin{gathered}
\mathbf{e}_{v}^{\gamma}=-\sin \gamma \cos \psi \mathbf{i}-\sin \gamma \sin \psi \mathbf{j}+\cos \gamma \mathbf{k}, \quad \mathbf{e}_{v}^{\psi}=-\cos \lambda \sin \psi \mathbf{i}+\cos \lambda \cos \psi \mathbf{j}, \\
\mathbf{e}_{v}^{\gamma} \perp \mathbf{e}_{v}, \mathbf{e}_{v}^{\psi} \perp \mathbf{e}_{v}, \mathbf{e}_{v}^{\gamma} \perp \mathbf{e}_{v}^{\psi} .
\end{gathered}
$$

The momentum equation is given by

$$
m \frac{d V}{d t} \mathbf{e}_{v}+m V\left(\frac{d \gamma}{d t} \mathbf{e}_{v}^{\gamma}+\frac{d \psi}{d t} \mathbf{e}_{v}^{\mu}\right)=(\rho \Pi-m) g \mathbf{k}-f_{d} \mathbf{e}_{v}+f_{l} \mathbf{e}_{l} .
$$

where $(m, \Pi)$ are the mass and volume of the rigid body; $\left(f_{d}, f_{l}\right)$ are the drag and lift forces.

Let $\mathbf{\Omega} *$ be the body's angular velocity, which is decomposed into two parts with the one along the unit vector $\mathbf{e}$ (self-spinning or bank) and the other part $\boldsymbol{\Omega}$ (azimuth and elevation) perpendicular to $\mathbf{e}$,

$$
\mathbf{\Omega}^{*}=\Omega_{s} \mathbf{e}+\Omega_{\omega}, \quad \mathbf{e}_{\omega} \cdot \mathbf{e}=0,
$$

where $\mathbf{e}_{\omega}$ is the unit vector of $\boldsymbol{\Omega}$ (perpendicular to $\mathbf{e}$ ),

$$
\mathbf{\Omega}=\Omega_{\omega} \quad \Omega=|\boldsymbol{\Omega}| .
$$

For axially symmetric body, $\mathbf{J}$ is a diagonal matrix

$$
\mathbf{J}=\left[\begin{array}{ccc}
J_{1} & 0 & 0 \\
0 & J_{2} & 0 \\
0 & 0 & J_{3}
\end{array}\right],
$$

with $J_{1}, J_{2}$, and $J_{3}$ the moments of inertia. The moment of momentum equation for small self-spinning velocity is given by (Chu and Fan, 2007) 


$$
\mathbf{J} \cdot \frac{d \boldsymbol{\Omega}}{d t} \approx \sigma \rho \Pi g \mathbf{e} \times \mathbf{k}+\sigma\left(f_{d} \mathbf{e} \times \mathbf{e}_{d}+f_{l} \mathbf{e} \times \mathbf{e}_{l}\right)+\mathbf{M}_{t}+\mathbf{M}_{a e} .
$$

where $\mathbf{M}_{a z}$ is the torque due to the azimuth and elevation rotation,

$$
\begin{gathered}
\mathbf{M}_{a e}=\frac{1}{2} \mathbf{e}_{\omega} \int_{-\frac{L}{2}}^{\frac{L}{2}} C_{r} \rho D\left(V_{r}-x \Omega\right)^{2} x d x=-\frac{1}{12} C_{r} \rho D L^{3} V_{r} \Omega \mathbf{e}_{\omega}, \quad \text { for } \frac{\Omega L}{2} \leq V_{r} \\
\mathbf{M}_{a e}=\mathbf{e}_{\omega}\left[\int_{-\frac{L}{2}}^{\frac{V_{r}}{2}} \frac{1}{2} C_{r} \rho D\left(V_{r}-x \Omega\right)^{2} x d x-\int_{\frac{V_{r}}{\Omega}}^{\frac{L}{2}} \frac{1}{2} C_{r} \rho D\left(V_{r}-x \Omega\right)^{2} x d x\right] \\
=-\frac{1}{2} C_{r} \rho D L^{2}\left[V_{r}^{2}\left(\frac{1}{4}-\left(\frac{V_{r}}{\Omega L}\right)^{2}\right)+\frac{4}{3} V_{r} \Omega L\left(\frac{V_{r}}{\Omega L}\right)^{3}+\frac{1}{2} \Omega^{2} L^{2}\left(\frac{1}{16}-\left(\frac{V_{r}}{\Omega L}\right)^{4}\right)\right] \mathbf{e}_{\omega} \\
\text { for } \frac{\Omega L}{2}>V_{r} .
\end{gathered}
$$

\section{Hydrodynamic coefficients}

Prediction of the rigid-body's orientation and COM location is to integrate the momentum equation (14) and moment of momentum equation (18) with known coefficients: $\mathrm{C}_{d}, C_{l}$, and $C_{m}$. Inner products between equation (14) and the unit vectors $\left(\mathbf{e}_{v}, \mathbf{e}_{v}^{\gamma}, \mathbf{e}_{v}^{\psi}\right)$ give

$$
\begin{gathered}
C_{d}=\frac{(\rho \Pi-m) g \mathbf{k} \cdot \mathbf{e}_{v}-m \frac{d V}{d t}}{\frac{1}{2} \rho A_{w}|V| V}, \\
C_{l}=\frac{m V\left(\mathbf{e}_{v}^{\gamma} d \gamma / d t+\mathbf{e}_{v}^{y} d \psi / d t\right) \cdot \mathbf{e}_{l}-(\rho \Pi-m) g \mathbf{k} \cdot \mathbf{e}_{l}}{\frac{1}{2} \rho A_{w}|V| V} .
\end{gathered}
$$

Inner product of (18) by the vector $\mathbf{e}_{r}$ leads to

$$
C_{m}=\frac{\mathbf{J} \cdot \frac{d \mathbf{\Omega}}{d t} \cdot \mathbf{e}_{r}-\sigma \rho \Pi g(\mathbf{e} \times \mathbf{k}) \cdot \mathbf{e}_{r}}{\frac{1}{2} \rho A_{w} L_{w} V^{2}}-\frac{\sigma}{L_{w}}\left(C_{d}\left(\mathbf{e} \times \mathbf{e}_{d}\right) \cdot \mathbf{e}_{r}+C_{l}\left(\mathbf{e} \times \mathbf{e}_{l}\right) \cdot \mathbf{e}_{r}\right),
$$

where

$$
\mathbf{e}_{r}=\mathbf{e}_{\omega} \times \mathbf{e}, \quad V_{r}=\mathbf{V} \cdot \mathbf{e}_{r} .
$$

The formulas (19)-(21) provide the basis for the experimental determination of $\left(\mathrm{C}_{d}, C_{l}, C_{m}\right)$ since each item in the right-hands of (19)-(21) can be measured by experiment. 


\section{Bomb drop experiment}

Models of Mk-84 bombs with and without tail section are taken as examples to illustrate the methodology for determination of the bulk drag/lift coefficients, and in turn the prediction of location and orientation of a fast-moving rigid-body through the water column. The primary objective is to determine the Mk84 trajectory through the very shallow water zone to provide an estimate of the maximum bomb-to-target standoff and required fuse delay time for optimum target lethality. Because it is possible that a portion, or all, of the guidance tail section may become separated from the warhead during water entry, it is necessary to determine the Mk84 trajectory for a variety of different tail configurations ranging from a warhead with a completely intact tail section and four fins to a warhead with the tail section completely.

Using the Hopkinson scaling laws, 1/12-scale Mk84 bomb models were designed and constructed in SRI that matched the overall casing shape and mass inertial properties of the full-scale Mk84 prototype. To model the different possible damaged tail configurations, we fabricated models that consisted of the warhead section with a complete tail section and four fins, a complete tail section and two fins, a complete tail section and no fins, and with the tail section removed. The models were accelerated to velocities of up to about $454 \mathrm{~m} \mathrm{~s}^{-1}$ using a gas gun. The gun was positioned over a $6 \mathrm{~m}$ deep by $9 \mathrm{~m}$ diameter pool, located at SRI's Corral Hollow Experiment Site (CHES). Two orthogonal Phantom 7 high-speed video (HSV) cameras operating at 10,000 fps were used to record the water entry and underwater trajectory. The digital HSV data were used to generate depth versus horizontal trajectory, position-time history, velocity-time history, deceleration-time history, and drag coefficient-time history profiles. Typically, up to three experiments were performed for each model configuration to determine the overall reproducibility (Gefken, 2006).

A gas gun with $0.10 \mathrm{~m}$ (4 in.) diameter and $1.52 \mathrm{~m}$ (60-in.) long was positioned over a $6.10 \mathrm{~m}(20-\mathrm{ft})$ deep by $9.14 \mathrm{~m}(30-\mathrm{ft})$ diameter pool located at SRI's Corral Hollow Experiment Site (CHES). The gas gun barrel was evacuated before launching the scale model to prevent an air blast from disturbing the water surface prior to the model impacting the water surface. At the end of the gas gun there was a massive steel ring to strip the sabot from the scale model. At high velocities there is some deviation from the theoretical calibration curve, which may be attributed to gas blow by around the sabot or friction. For the maximum gun operating pressure of 2,500 psi, we were able to achieve a nominal water-entry velocity of about $304.80 \mathrm{~m} / \mathrm{s}$.

Two orthogonal periscope housings were positioned in the pool to allow simultaneous above-water and below-water visualization of the model trajectory. The housings supported Phantom 7 high-speed video (HSV) cameras, which were run at 10,000 fps. Five high-intensity, short duration $(30 \mathrm{~ms})$ flash bulbs were used to front-light the scale model as it entered the water and traveled under water. The HSV cameras and flash bulbs were triggered at the time the sabot was released within the gun. 
A series of 19 experiments was performed with the different 1/12-scale Mk84 bomb models described in subsection 6.2 with nominal water-entry velocities ranging from $119.48 \mathrm{~m} / \mathrm{s}$ to $308.83 \mathrm{~m} / \mathrm{s}$. Table 1 summarizes the overall experimental matrix and water-entry conditions. Typically, the water-impact angle of entry was between $88^{\circ}$ and $90^{\circ}$. In Experiments 10, 11, and 12 the sabot failed to fully support the scale model within the gun during the launch phase, resulting in the scale model impacting the sabot stripper plate before impacting the water. All the experimental data have been converted to full-scale values.

Table 1: $\quad$ Summary of Mk84 underwater trajectory experimental matrix.

\begin{tabular}{|l|l|l|l|}
\hline $\begin{array}{l}\text { Experiment } \\
\text { Number }\end{array}$ & Model Type & $\begin{array}{l}\text { Water-Entry } \\
\text { Velocity }(\mathrm{m} / \mathrm{s})\end{array}$ & $\begin{array}{l}\text { Water-Entry } \\
\text { Impact Angle }\left(^{\circ}\right)\end{array}$ \\
\hline 1 & I & 131.51 & 89.2 \\
\hline 2 & I & 296.87 & 90.0 \\
\hline 3 & I & 295.35 & 88.8 \\
\hline 4 & I & 302.05 & 88.5 \\
\hline 5 & I & 226.77 & 88.0 \\
\hline 6 & I & 219.45 & 89.0 \\
\hline 7 & I & 119.48 & 88.2 \\
\hline 8 & II Model & impacted sabot & stripper plate \\
\hline 9 & II Model & impacted sabot & stripper plate \\
\hline 10 & II & 295.04 & 90.0 \\
\hline 11 & II & 289.96 & 90.0 \\
\hline 12 & II Model & impacted sabot & stripper plate \\
\hline 13 & IV & 296.26 & 85.7 \\
\hline 14 & IV & 300.53 & 90.0 \\
\hline 15 & IV & 300.53 & 88.7 \\
\hline 16 & III & 304.19 & 90.0 \\
\hline 17 & III & 298.39 & 87.0 \\
\hline 18 & III & 291.08 & 88.1 \\
\hline 19 & II & 296.87 & 90.0 \\
\hline
\end{tabular}

\section{Semi-empirical formulas}

Upon completion of the drop phase, the video from each camera was converted to digital format. For Mk84 warhead without tail section, vertical and horizontal locations of the two-end points (Fig. 2) versus time were recorded. From these data, the unit vector e can be directly determined using (1b). The translation velocity $\mathbf{u}$ and the angular velocity $\mathbf{\Omega}$ are measured and so as the fluid-to-body relative velocity $\mathbf{V}$ since it is assumed that the water velocity is much smaller than the bomb velocity [i.e., (2b) holds]. The unit vectors $\left(\mathbf{e}_{v}, \mathbf{e}_{l}\right)$ are in turned determined since $\left(\mathbf{e}_{v}, \mathbf{e}_{l}\right)$ represent the direction of $\mathbf{V}$ and its $90^{\circ}$ shift. When the orientation of the bomb is measured, the unit vector $\mathbf{e}$ is known and so as $\mathbf{e}_{\omega}$ using (22). 


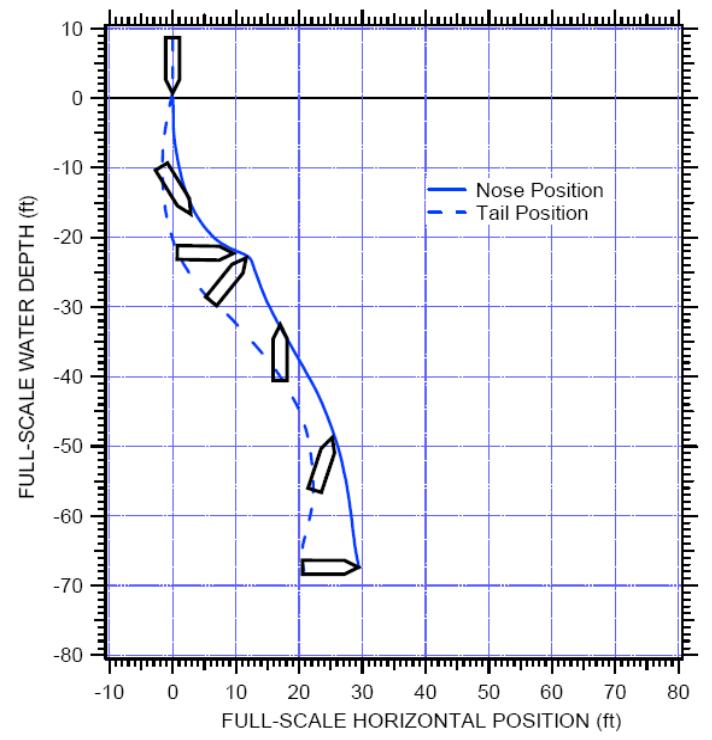

Figure 2: $\quad$ Trajectory of Mk84 with no tail section and water-entry velocity of 296 m/s (Exp-13) (from Gefken, 2006).

Using the unit vector $\mathbf{e}_{v}$, we determine the elevation angle $\gamma$ and the azimuth angle $\psi\left[\right.$ see (10)] and the two other unit vectors $\left(\mathbf{e}_{v}^{\gamma}, \mathbf{e}_{v}^{\psi}\right)$ [see (11)]. Using the unit vectors $\mathbf{e}$ and $\mathbf{e}_{\omega}$, we determine the unit vector $\mathbf{e}_{\mathrm{r}}$ [see (22)]. With the calculated temporally varying $\left(\mathrm{C}_{d}, \mathrm{C}_{l}, C_{m}\right)$ and $(\alpha, \mathrm{Re})$ data, we obtain the following semi-empirical formulas for calculating the hydrodynamic coefficients,

$$
\begin{gathered}
C_{d}(\operatorname{Re}, \alpha)=\left\{\begin{array}{ll}
8 \sin (2 \alpha)\left(\frac{\mathrm{Re}_{\text {crit }}}{\mathrm{Re}}\right)^{2}+0.02 & \text { if } \sin (2 \alpha) \geq 0 \\
0.34|\sin (2 \alpha)|\left(\frac{\mathrm{Re}_{c r i t}}{\mathrm{Re}}\right)+0.02 & \text { and } \mathrm{Re} \geq \mathrm{Re}_{\text {crit }},
\end{array},\right. \\
C_{l}(\mathrm{Re}, \alpha)= \begin{cases}2.5 \sin (2 \alpha) \min \left[\left(\frac{\mathrm{Re}}{\mathrm{Re}_{c r i t}}\right)^{1.2},\left(\frac{\mathrm{Re}_{c r i t}}{\mathrm{Re}}\right)^{1.2}\right] & \text { if } \sin (2 \alpha) \geq 0 \\
0.16 \sin (2 \alpha) & \text { if } \sin (2 \alpha)<0\end{cases} \\
\operatorname{Re}_{\text {crit }}=1.5 \times 10^{7},
\end{gathered}
$$




$$
C_{m}=A \sin \theta-B \frac{d \alpha}{d t}, A=\left\{\begin{array}{ll}
0.06 \quad \text { if } \theta \leq 180 \\
0.006 & \text { if } \theta>180
\end{array}, B=0.00065\right.
$$

\section{Verification}

The semi-empirical formulas of $\left(C_{d}, C_{l}, C_{m}\right)$ were verified using the data collected from the experiments. We use the formulas (33)-(35) to compute the hydrodynamic coefficients $\left(C_{d}, C_{l}, C_{m}\right)$, and then to predict the location and orientation of Mk-84 bomb in the water column by (14) and (18). Comparison between model predictions and experiments (Fig. 3) shows the validity of feasibility of the semi-empirical formulas (33)-(35).
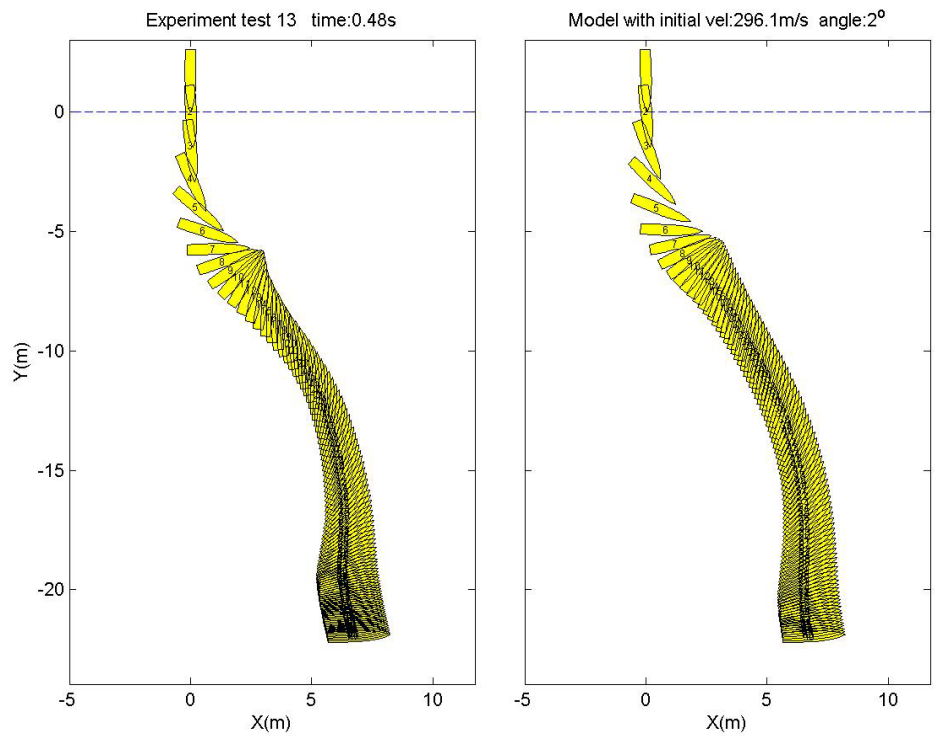

Figure 3: Comparison between modelled and observed bomb trajectories for the experiment shown in Fig. 2.

\section{Conclusions}

A new method has been developed to determine the hydrodynamic coefficients $\left(\mathrm{C}_{d}, \mathrm{C}_{l}, C_{m}\right)$ of fast-moving rigid body in the water column. This method contains two parts: (1) establishment of the inverse relationship between $\left(\mathrm{C}_{d}, \mathrm{C}_{l}, C_{m}\right)$ and the rigid-body's trajectory and orientation, and (2) experiments for collecting data of the rigid-body's trajectory and orientation. Using the experimental data, the inverse relationship leads to semi-empirical formulas of $\left(\mathrm{C}_{d}, \mathrm{C}_{l}, C_{m}\right)$ versus Reynolds number and attack angle. This method is much cheaper than the 
traditional one using the wind tunnel to determine $\left(\mathrm{C}_{d}, \mathrm{C}_{l}, C_{m}\right)$. We also verify these formulas using the experimental data.

\section{Acknowledgement}

The Office of Naval Research Breaching Technology Program and Naval Oceanographic Office supported this study.

\section{References}

[1] Chu, P.C., C.W. Fan, A. D. Evans, and A. Gilles, 2004: Triple coordinate transforms for prediction of falling cylinder through the water column. Journal of Applied Mechanics, 71, 292-298.

[2] Chu, P.C., A. Gilles, and C.W. Fan, 2005a: Experiment of falling cylinder through the water column. Experimental and Thermal Fluid Sciences, 29, 555-568.

[3] Chu, P.C., and C.W. Fan, 2005b: Pseudo-cylinder parameterization for mine impact burial prediction. Journal of Fluids Engineering, 127, 1515-152.

[4] Chu, P.C., and C.W. Fan, 2006a: Prediction of falling cylinder through airwater-sediment columns. Journal of Applied Fluid Mechanics, 73, 300-314.

[5] Chu, P.C., and G. Ray, 2006b: Prediction of high speed rigid body maneuvering in air-water-sediment columns, Advances in Fluid Mechanics, 6, 123-132.

[6] Chu, P.C., and C.W. Fan, 2007: Mine impact burial model (IMPACT35) verification and improvement using sediment bearing factor method. IEEE Journal of Oceanic Engineering, 32 (1), 34-48.

[7] Gefken, P., 2006: Evaluation of precision-guided bomb trajectory through water using scaled-model experiments. SRI Final Technical Report-PYU16600, Menlo Park, California, USA, 1-80. 